Erich J. Muehlegger, Andrew S. Raich, Cesar E. Silva, Wenhuan Zhao, Department of Mathematics, Williams College, Williamstown, Massachusetts 01267, e-mail:csilva@williams.edu

\title{
LIGHTLY MIXING ON DENSE ALGEBRAS
}

\begin{abstract}
We use a set in the unit interval to construct measure preserving transformations that are lightly mixing on intervals but not ergodic, and ergodic measure preserving transformations that are lightly mixing on intervals but not lightly mixing, and give some applications.
\end{abstract}

\section{Introduction}

Let $X$ denote the unit interval $[0,1]$ and $\mu$ Lebesgue measure. A transformation is a map $T: X \rightarrow X$ that is one-to-one and onto a.e. such that $T$ and $T^{-1}$ are measurable. $T$ is measure preserving if for all measurable sets $A$, $\mu(T A)=\mu(A) . T$ is ergodic if for any measurable set $A, T(A)=A$ implies $\mu(A)=0$ or 1 . We identify sets that differ by a null set and write $A=B$ to mean $\mu(A \triangle B)=0$.

Let $s=\left\{n_{k}\right\}$ be an infinite sequence. A finite measure preserving transformation $T$ sweeps out on $s$ if for all sets $A$ of positive measure, $\bigcup_{k=0}^{\infty} T^{n_{k}} A=X$. Since $X$ has finite measure, ergodicity is equivalent to the property that for all sets $A$ of positive measure, $T$ sweeps out on cofinite sequences. $T$ is lightly mixing if for all sets $A$ and $B$ of positive measure

$$
\liminf _{n \rightarrow \infty} \mu\left(T^{n} A \cap B\right)>0 .
$$

It was shown in [2] that $T$ sweeps out on all sequences if and only if $T$ is lightly mixing. King [7] showed that the Cartesian product of lightly mixing transformations is lightly mixing, answering a question of Friedman; the question arises from [7] of whether there is a weak mixing transformation $T$ such that $T$ satisfies the lightly mixing condition on a dense algebra but is

Key Words: Ergodic, lightly mixing, dense algebra

Mathematical Reviews subject classification: 28D05

Received by the editors November 22, 1996 
not lightly mixing. $T$ is said to be lightly mixing on an algebra $\mathcal{A}$ if for all $A \in \mathcal{A}$ of positive measure and all measurable sets $B$ of positive measure, $\liminf _{n \rightarrow \infty} \mu\left(T^{n} A \cap B\right)>0$. The same proof in [2] shows that $T$ is lightly mixing on an algebra if and only if, for each set in the algebra, $T$ sweeps out on all sequences.

In this paper we construct an ergodic transformation $V$ such that $V$ is lightly mixing on intervals but is not lightly mixing. We first see this as an application of the properties of a subset $K^{*}$ of the reals based on the Cantor set. The transformation that we construct could be based on any lightly mixing transformation of the interval, but to make our construction more explicit we use a specific transformation of Chacon [3] that has been shown to be lightly mixing in [6]. We also obtain a finite measure preserving transformation $T$ that is not ergodic but is lightly mixing on intervals (and uniformly sweeping out on intervals). We conclude with a result that abstracts the main properties of our example using the notion of singular measures.

The transformation $V$ above satisfies the even stronger condition of being uniformly sweeping out on intervals (while not being uniformly sweeping out). A transformation $T$ is said to be uniformly sweeping out if for all $\epsilon>0$ and each set $A$ of positive measure there exists an integer $N=N(\epsilon, A)$ such that $\mu\left(\bigcup_{i=1}^{N} T^{k_{i}}(A)\right)>1-\epsilon$, for all sequences $k_{1}<k_{2}<\cdots<k_{N}$. (King [8] has also shown that Cartesian products of uniformly sweeping out transformations are uniformly sweeping out.) However, it is well-known that other properties such as mixing on a sequence $\left(\lim _{i \rightarrow \infty} \mu\left(T^{n_{i}} A \cap B\right)=\mu(A) \mu(B)\right.$, for some sequence $\left\{n_{i}\right\}$ ), and hence mixing, carry over from a dense algebra to the generated sigma algebra. It is known that the properties of mixing, uniform sweeping out, light mixing, mild mixing and weak mixing are in strictly decreasing order, cf. [1], [4], [6] and the references therein.

Acknowledgments. This paper is based on research in the Dynamics Group of the 1996 SMALL undergraduate summer research project at Williams College with Prof. C. Silva as faculty advisor. The group started by reading Friedman [5] on constructions in ergodic theory. A question the group considered was what strong conditions a non-ergodic measure preserving transformation could satisfy on a dense algebra; this led to this work. Support for the project was provided by a National Science Foundation REU grant and the Bronfman Science Center of Williams College.

We would like to thank Professor Terrence Adams for suggesting that we use the set $K^{*}$ in the construction of the transformations. We are indebted to the referees for several remarks that improved the exposition and for the formulation of Theorem 2.3. 


\section{Construction of the Set $K^{*}$}

We construct a set $K^{*}$ that will be used to define our transformations. This set is known and has been used in ergodic theory in a different context in Maharam [9] to construct an ergodic measure preserving transformation which is not continuous when restricted to the complement of any null set. While the construction is standard we present it here for completeness.

Let $K$ be a Cantor set of measure $1 / 2$ in $X$. Given an open interval $I$ in $X$, define a modified Cantor set, denoted by $K(I)$, in the following way. Let $K^{\prime}=K \backslash\{0,1\}$. Scale the set $K^{\prime}$ by the factor $\mu(I)$ and place the scaled copy at the left end point of $I$. Denote the new set by $K(I)$.

Now proceed iteratively. Let $L_{1}=K((0,1 / 2))$ and $L_{1}^{*}=K((1 / 2,1))$. Assume $L_{n-1}$ and $L_{n-1}^{*}$ have been defined. The complement in $(0,1 / 2)$ or $(1 / 2,1)$ of each of these sets consists of a countable disjoint collection of open intervals denoted $A_{i}$ and $B_{i}$, respectively. If $n$ is even let $L_{n}^{*}=\bigcup_{i=1}^{\infty} K\left(A_{i}\right)$ and $L_{n}=\bigcup_{i=1}^{\infty} K\left(B_{i}\right)$. If $n$ is odd let $L_{n}=\bigcup_{i=1}^{\infty} K\left(A_{i}\right)$ and $L_{n}^{*}=\bigcup_{i=1}^{\infty} K\left(B_{i}\right)$.

Finally define

$$
K^{*}=\bigcup_{n=1}^{\infty} L_{n}
$$

Note that $\bigcup_{n=1}^{\infty} L_{n}^{*} \subset K^{* c}$. The proof of the following proposition is left to the reader.

Proposition 2.1. Given an interval $I \subseteq[0,1], \mu\left(I \cap K^{*}\right)>0$ and $\mu\left(I \cap K^{* c}\right)>$ 0 .

Since $K^{*}$ is a Borel set, it is well-known that it is isomorphic to an interval. In the proposition below we make this more explicit with a standard function.

Proposition 2.2. The functions $\phi: K^{*} \rightarrow[0,1 / 2]$ and $\psi: K^{* c} \rightarrow[0,1 / 2]$ defined by $\phi(x)=\mu\left(K^{*} \cap[0, x)\right)$ and $\psi(x)=\mu\left(K^{* c} \cap[0, x)\right)$ have well-defined inverses a.e. and are measure preserving.

\section{The Transformations}

Our constructions can be based on any lightly mixing transformation on the interval. However, to be more explicit we present a finite measure preserving transformation defined by Chacon in [3] and shown to be lightly mixing by Friedman and King [6]. Later it was shown in Adams [1] that the transformation satisfies the stronger property of being uniformly sweeping out. We modify the original transformation, defining it on the interval $[0,1 / 2]$ instead of the interval $[0,1)$. We first define a sequence of columns $\left\{C_{n}\right\}$, where each 
$C_{n}$ consists of finitely many intervals. Let $C_{1}=\left\{\left[0, \frac{1}{4}\right)\right\}$. To obtain $C_{2}$, cut the interval in $C_{1}$ into the intervals $[0,1 / 8),[1 / 8,1 / 4)$, stack the right half on top of the left half, and put the interval $[1 / 4,3 / 8)$ on top. We call this new interval a spacer.

In general, to obtain column $C_{n+1}$ from $C_{n}$, cut the intervals in the column $C_{n}$ in half, stack them from left to right and add the spacer $\left[1 / 2-\frac{1}{2^{n+1}}, 1 / 2-\right.$ $\frac{1}{2^{n+2}}$ ), on top of all the intervals. The spacer placed above $C_{n+1}$ has the same measure as the right half of a level in $C_{n}$. All the intervals in a column have the same length. Thus $\lim _{n \rightarrow \infty} \mu\left(C_{n}\right)=\frac{1}{4} \cdot \sum_{i=0}^{\infty} \frac{1}{2^{i}}=1 / 2$.

Let $I_{n, i}$ denote the $i$ th interval of $C_{n}$ starting at the bottom for $1 \leq i \leq$ $h_{n}$, where $h_{n}$ is the number of intervals in the column $C_{n}$. Now define a transformation $S$ to map each point of $I_{n, i}$ to the point directly above it on level $I_{n, i+1}$ (use the translation that sends an interval to another). This defines the transformation on the interval $[0,1 / 2)$; we let $S(1 / 2)=1 / 2$.

Now define two transformations, $T_{1}: K^{*} \rightarrow K^{*}$ and $T_{2}: K^{* c} \rightarrow K^{* c}$ by $T_{1}=\phi^{-1} \circ S \circ \phi$ and $T_{2}=\psi^{-1} \circ S \circ \psi$. Then both $T_{1}$ and $T_{2}$ are clearly finite measure preserving and lightly mixing on $K^{*}$ and $K^{* c}$, respectively.

Proposition 3.1. There exists a finite measure preserving transformation $T$ that is not ergodic but is lightly mixing on intervals.

ProOF. Let

$$
T(x)= \begin{cases}T_{1}(x), & \text { for } x \in K^{*}, \\ T_{2}(x), & \text { for } x \in K^{* c} .\end{cases}
$$

For convenience, given any set of positive measure $A$ write $A_{1}=A \cap K^{*}$ and $A_{2}=A \cap K^{* c}$. If $I$ is an interval in [0,1], by Proposition $1.1, \mu\left(I \cap K^{*}\right)>0$ and $\mu\left(I \cap K^{* c}\right)>0$. One can verify that

$$
T^{n} I \cap A=\left(T_{1}^{n} I_{1} \cap A_{1}\right) \cup\left(T_{2}^{n} I_{2} \cap A_{2}\right)
$$

Since $T_{1}$ and $T_{2}$ are lightly mixing and either $\mu\left(A_{1}\right)>0$ or $\mu\left(A_{2}\right)>0$ we have that

$$
\liminf _{n \rightarrow \infty} \mu\left(T^{n} I \cap A\right)>0 .
$$

As $T K^{*}=K^{*}, T$ is not ergodic.

Remark 3.1. a) There exist ergodic finite measure preserving transformations for which there is no algebra on which the transformation is lightly mixing. In fact, this is true for any rigid transformation such as a rotation by an irrational angle. Rigid transformations satisfy the property that for any set $A$ of positive measure, $\liminf _{n \rightarrow \infty} \mu\left(T^{n} A \cap A^{c}\right)=0$. 
b) One can consider the weaker notion of lightly mixing between intervals: for all intervals $I, J$, $\liminf _{n \rightarrow \infty} \mu\left(T^{n} I \cap J\right)>0$. If in the proof of Proposition 2.1 we choose $T_{1}$ to be lightly mixing and $T_{2}$ to be not lightly mixing, then $T$ will be lightly mixing between intervals but will not sweep out on all sequences for intervals, hence $T$ will not be lightly mixing on the algebra of intervals.

To define our next transformation we need two transformations $V_{1}: K^{*} \rightarrow$ $K^{* c}$ and $V_{2}: K^{* c} \rightarrow K^{*}$ defined by $V_{1}=\psi^{-1} \circ S \circ \phi$ and $V_{2}=\phi^{-1} \circ S \circ \psi$.

Theorem 3.2. Let $V:[0,1] \rightarrow[0,1]$ be the measure preserving transformation defined by

$$
V(x)= \begin{cases}V_{1}(x), & \text { for } x \in K^{*}, \\ V_{2}(x), & \text { for } x \in K^{* c}\end{cases}
$$

Then $V$ is ergodic and lightly mixing on intervals but is not totally ergodic, hence not lightly mixing.

Proof. Let $I$ be an interval and $A$ be a set of positive measure. We will show that

$$
\liminf _{n \rightarrow \infty} \mu\left(V^{n} I \cap A\right)>0 .
$$

If $\mu\left(A \cap K^{*}\right)>0$ let $A^{\prime}=A \cap K^{*}$. If $\mu\left(A \cap K^{*}\right)=0$, a similar argument follows by letting $A^{\prime}=A \cap K^{* c}$. Then,

$$
\begin{aligned}
& \liminf _{n \rightarrow \infty} \mu\left(V^{n} I \cap A\right) \\
& \quad \geq \min \left\{\liminf _{n \rightarrow \infty} \mu\left(V^{(2 n)} I \cap A^{\prime}\right), \liminf _{n \rightarrow \infty} \mu\left(V^{(2 n+1)} I \cap A^{\prime}\right)\right\} \\
& \quad \geq \min \left\{\liminf _{n \rightarrow \infty} \mu\left(V^{(2 n)}\left(I \cap K^{*}\right) \cap A^{\prime}\right), \liminf _{n \rightarrow \infty} \mu\left(V^{(2 n)}\left(V(I) \cap K^{*}\right) \cap A^{\prime}\right)\right\}
\end{aligned}
$$

Now we observe that

$$
V^{2}(x)= \begin{cases}\phi^{-1} \circ S^{2} \circ \phi(x), & \text { for } x \in K^{*}, \\ \psi^{-1} \circ S^{2} \circ \psi(x), & \text { for } x \in K^{* c}\end{cases}
$$

Since $S^{2}$ is lightly mixing both terms above on the right hand side are positive, and therefore $V$ is lightly mixing on intervals.

To show $V$ is ergodic it suffices to show that for any $B \subset[0,1)$ where $\mu\left(B \cap K^{*}\right)>0, B$ sweeps out. If $\mu\left(B \cap K^{* c}\right)>0$, the argument is similar. 
Now,

$$
\begin{aligned}
\bigcup_{k=1}^{\infty} V^{k} B & =\left(\bigcup_{k \text { even }} V^{2 \frac{k}{2}}(B)\right) \bigcup\left(\bigcup_{k \text { odd }} V \circ V^{2 \frac{(k-1)}{2}}(B)\right) \\
& =\phi^{-1}([0,1 / 2]) \cup V\left(\phi^{-1}([0,1 / 2])\right)=K^{*} \cup K^{* c} \\
& =X .
\end{aligned}
$$

Finally we note that $V^{2} K^{*}=K^{*}$.

Remark 3.2. Since $S^{2}$ is uniformly sweeping out [1], using a similar technique as in Theorem 2.2 one can show that $V$ is uniformly sweeping out on intervals but not uniformly sweeping out. Since intervals satisfy a strong property, but the transformation is not totally ergodic this provides the seed for various counterexamples.

If we define $\nu(A)=\mu\left(A \cap K^{*}\right) / \mu\left(K^{*}\right)$ and $\gamma(B)=\mu\left(B \cap K^{* c}\right) / \mu\left(K^{* c}\right)$ then $\nu$ and $\gamma$ are mutually singular measures on $X$, (i.e., there exist disjoint sets $C$ and $D$ whose union is $X$ and such that $\nu(C)=\gamma(D)=0)$, and $(V, \nu)$ and $(V, \gamma)$ are finite measure preserving lightly mixing transformations. The following result captures the main idea of the examples, and its proof is as before.

Theorem 3.3. Let $S: Y \rightarrow Y$ be the shift transformation on the space $Y$ of all infinite sequences of 0 's and 1 's. Let $\nu$ be the product measure $(1 / 3,2 / 3)$ and $\gamma$ the product measure $(2 / 3,1 / 3)$ on $Y$, and $\mu=1 / 2 \nu+1 / 2 \gamma$. Let $\theta: Y \rightarrow Y$ be the flip that switches 0 's and 1 's. If we define $T$ by $T=S \circ \theta$ then $T$ is an ergodic finite measure preserving transformation that is lightly mixing on the algebra of cylinder sets and has a 2-point rotation factor.

\section{References}

[1] T. Adams, Uniformly sweeping out does not imply mixing, Illinois J. Math., 37 (1993), 525-536.

[2] J. R. Blum, S. L. M. Christianson and D. Quiring, Sequence mixing and $\alpha$-mixing, Illinois J. Math., 18 (1974), 131-135.

[3] R. V. Chacon, Weakly mixing transformations which are not strongly mixing, Proc. Am. Math. Soc., 22 (1969), 559-562.

[4] N. A. Friedman, Mixing and sweeping out, Israel J. Math 68 (1989), 365-375. 
[5] N. A. Friedman, Replication and stacking in ergodic theory, Amer. Math. Month. 99 (1992), 31-41.

[6] N. A. Friedman and J. L. King, Rank one lightly mixing, Israel J. Math 73 (1991), 281-288.

[7] J. L. King, Lightly mixing is closed under countable products, Israel J. Math 62 (1988), 341-346.

[8] J. L. King, Closure properties for the class of uniformly sweeping-out transformations, Illinois J. Math 36 (1992), 233-237.

[9] D. Maharam, On orbits under ergodic measure-preserving transformations, Trans. Amer. Math. Soc. 119 (1965), 51-66. 